\title{
Translation of the UK Pediatric Influenza Vaccination Programme in Primary Schools to 13 European Countries Using a Dynamic Transmission Model
}

\section{Laetitia Gerlier ${ }^{1}$, Judith Hackett ${ }^{2}$, Richard Lawson ${ }^{2}$, Sofia Dos Santos Mendes ${ }^{3}$, Martin Eichner ${ }^{4,5}$}

${ }^{1}$ QuintilesIMS, Real-World Evidence Solutions, Zaventem, Belgium

${ }^{2}$ AstraZeneca, Gaithersburg, MD, USA

${ }^{3}$ AstraZeneca, Brussels, Belgium (Current affiliation: MSD, Brussels, Belgium)

${ }^{4}$ Institute for Clinical Epidemiology and Applied Biometry, University of Tübingen, Tübingen, Germany ${ }^{5}$ Epimos GmbH, Dusslingen, Germany

For correspondence: Laetitia.Gerlier@quintilesims.com

\section{Abstract}

Objectives: To simulate the impact of a pediatric influenza vaccination programme using quadrivalent live attenuated influenza vaccine (QLAIV) in Europe by applying coverage rates achieved in the United Kingdom during the 2014-2015 season and to compare the model outcomes to the UK results.

Methods: We used a deterministic, age-structured, dynamic transmission model adapted to the demography, contact patterns and influenza incidence of 13 European countries, with a 10-year horizon. The reference strategy was the unchanged country-specific coverage rate, using quadrivalent inactivated vaccine (assumed efficacy against infection from $45 \%$ in 1 -year-old children to $60 \%$ in healthy adults). In the evaluated strategy, $56.8 \%$ of $5-10$-year-old children were additionally vaccinated with QLAIV (assumed efficacy 80\%), as was the case in 2014-2015 in the United Kingdom's primary school pilot areas. Symptomatic influenza cases and associated medical resources (primary care consultations [PCC], hospitalization, intensive care unit [ICU] admissions) were calculated. The evaluated versus reference strategies were compared using odds ratios (ORs) for PCC in the target (aged 5-10-years) and non-target adult (aged >17 years) populations as well as number needed to vaccinate (NNV) with QLAIV to avert one PCC, hospitalization or ICU admission. Model outcomes, averaged over 10 seasons, were compared with published real-life data from the United Kingdom for the 20142015 season.

Results: Over 13 countries and 10 years, the evaluated strategy prevented 32.8 million of symptomatic influenza cases (172.3 vs 205.2 million). The resulting range of ORs for PCC was $0.18-0.48$ among children aged 5-10-years, and the published OR in the United Kingdom was 0.06 (95\% confidence interval [0.01; 0.62]). In adults, the range of ORs for PCC was 0.60-0.91 (UK OR=0.41 [0.19;0.86]). NNV ranges were 6-19 per averted PCC (UK NNV=16), 530-1524 per averted hospitalization (UK NNV=317) and 5298-15 241 per averted ICU admission (UK NNV=2205).

Conclusions: Across a range of European countries, our model shows the beneficial direct and indirect impact of a paediatric vaccination programme using QLAIV in primary school-aged children, consistent with what was observed during a single season in the United Kingdom. Recommendations for the implementation of pediatric vaccination programmes are, therefore, supported in Europe.

Keywords: seasonal influenza, pediatric vaccination, live-attenuated influenza vaccine, indirect protection, dynamic transmission model, Europe 


\section{INTRODUCTION}

Among countries that have implemented an influenza vaccination programme including children (Canada, Finland, United Kingdom, United States), two main indicators are used to assess the performance of the newly implemented vaccination campaign: the coverage rate reached in the target population and the number of prevented clinical events in the total population. The former outcome can be estimated via surveys or administrative methods, ${ }^{1}$ while the latter implies a comparison of the number of clinical events before and after changing the coverage rate. Canadian and US studies analyzing a sufficiently long period of time demonstrated substantial reduction of the influenza-related morbidity through a universal influenza vaccination programme including healthy children., ${ }^{2,3}$ Other studies focusing on a single influenza season demonstrated both direct benefits of pediatric vaccination in the target-population and indirect benefits in the general population..$^{4-8} \mathrm{~A}$ systematic literature review confirmed that the vaccination of healthy children against influenza provides both health benefits to the children themselves and economic benefits to the community. ${ }^{9}$ The authors however highlight the difficulty in measuring these effects, and the need for further research. As a complement to observational studies, modelling exercises were also useful to compare different vaccination stategies. Dynamic transmission models, considering direct and indirect protection in a population, generally emphasized large protection effects, ${ }^{10-13}$ yet the question must be addressed whether the magnitude of protective effects is reflective of real life as transmission models must invariably be based on simplifying assumptions. In our study, we used a dynamic transmission model available for 13 European countries ${ }^{1,14-18}$ to simulate the UK 2014-2015 pediatric vaccination programme and compare model outcomes to observed, real-life results.

\section{AIMS AND OBJECTIVES}

We simulate the impact of a pediatric influenza vaccination programme using a quadrivalent live attenuated influenza vaccine (QLAIV) by applying coverage rates and outcomes achieved in the United Kingdom during the 2014-2015 season to 13 European countries, including Austria, Belgium, Finland, France, Germany, Greece, Italy, Luxembourg, The Netherlands, Poland, Portugal, Spain, and Sweden. A secondary objective was to compare the direct and indirect impact of the vaccination programme from the model with the UK results.

\section{METHODS}

\section{Study Design}

A deterministic, age-structured, dynamic transmission model was used to simulate the transmission of influenza in the population and to compare different vaccination strategies including direct and indirect protection effects. The model simulates the independent transmission of four influenza viruses strains: $A(H 1 N 1)$, $\mathrm{A}(\mathrm{H} 3 \mathrm{~N} 2)$, one B strain coming from the B/Victoria lineage, and one B strain coming from the B/Yamagata lineage. Demographic changes and transmission dynamics are described by a system of interacting differential equations. Contact patterns between individuals (ie, average age-dependent numbers of contacts per person per day) were derived from the European Polymod study, using the matrix for physical and non-physical contacts. ${ }^{19}$ The all-year average of the basic reproduction number $\mathrm{R}_{0}$, representing the number of secondary infections produced by a single infected case in a fully susceptible population, was calibrated to country-specific reported incidence data for laboratory-confirmed influenza, averaged over two or more seasons. $\mathrm{R}_{0}$ was assumed to vary throughout the year: it was $43 \%$ higher than average around Christmas and $43 \%$ lower in summer. ${ }^{11}$ Model inputs and assumptions are presented in Table 1 (values common to all countries) and Table 2 (country-specific values). Further details on the model design and methods were described in a previously published study. ${ }^{16}$ 


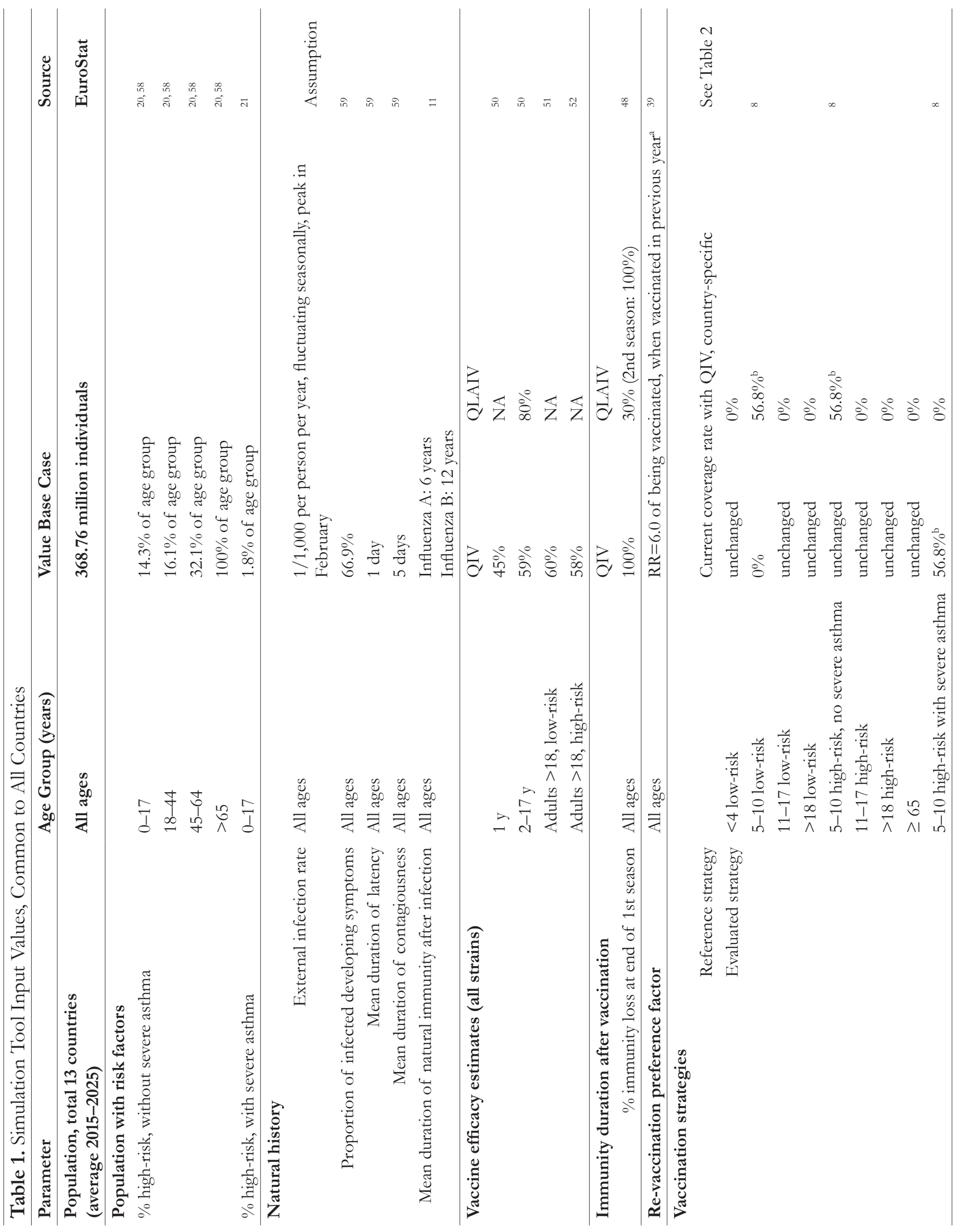




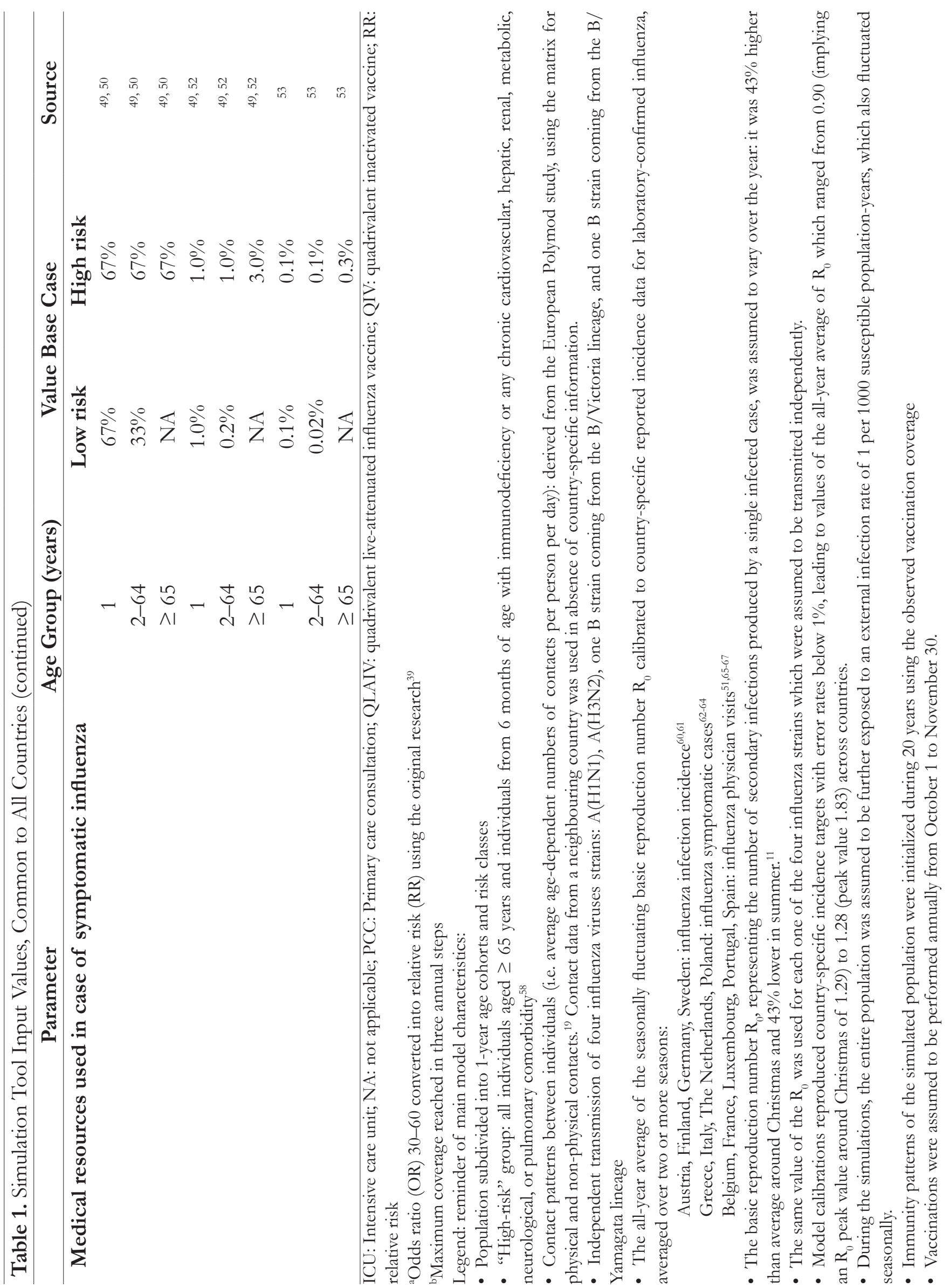




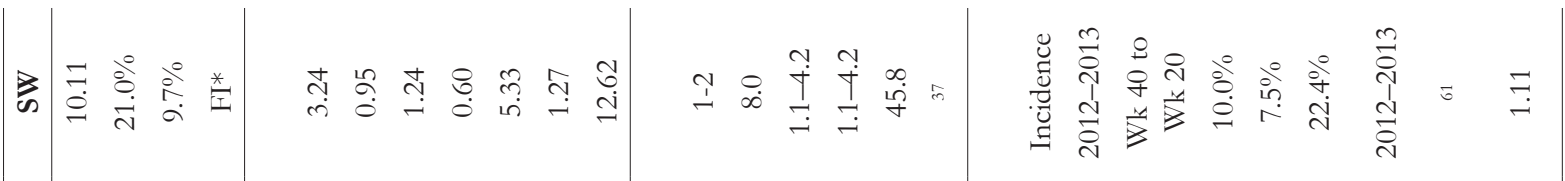

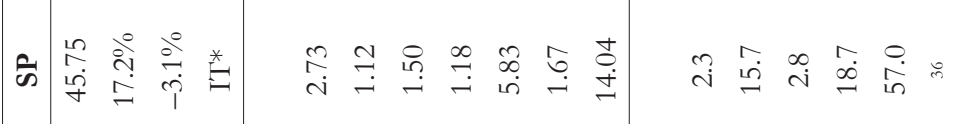

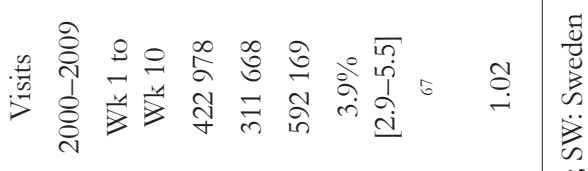

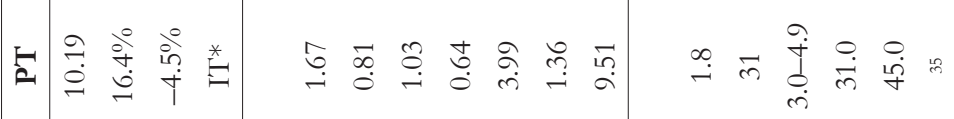

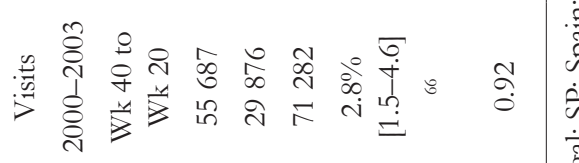

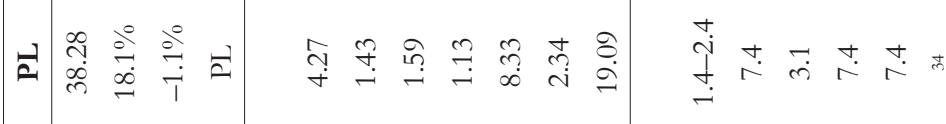

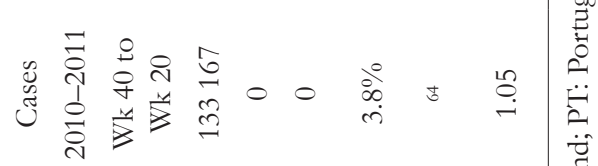

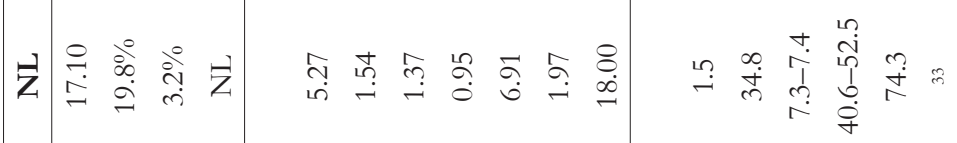

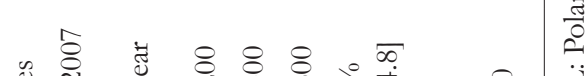

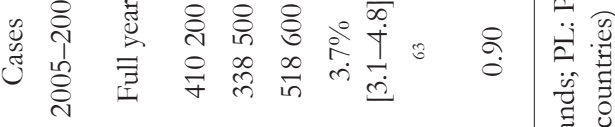

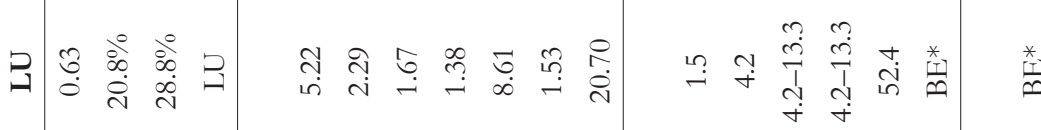

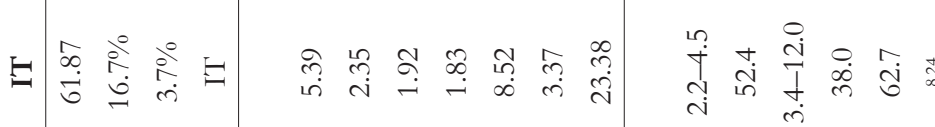

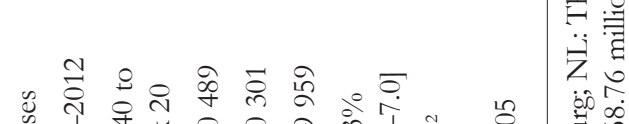

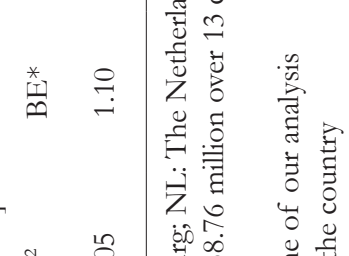

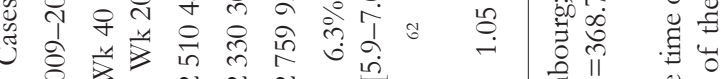

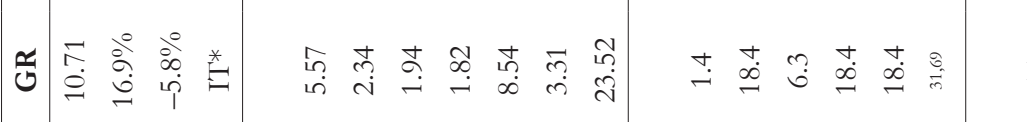

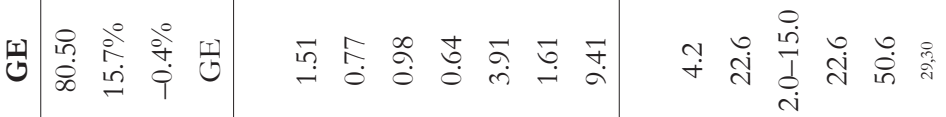

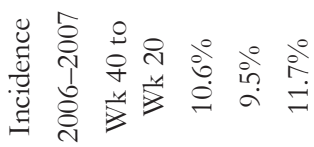

$\square$
$\square$

$+$

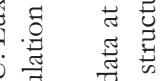

:

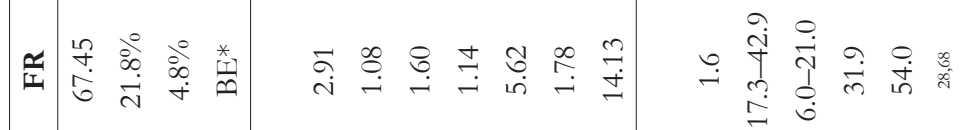

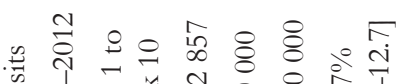

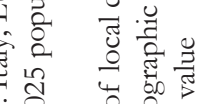

ᄀ

苞苞䓂

ชิ ส

㲾

(

प

$\frac{\sqrt[3]{3}}{\pi}$

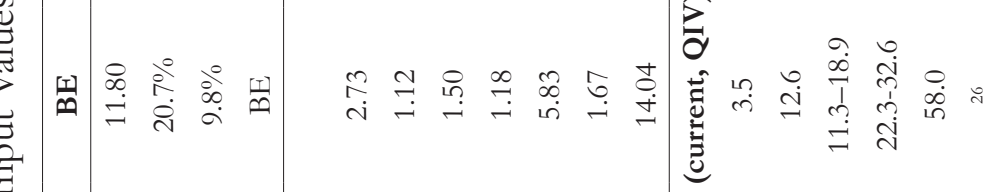

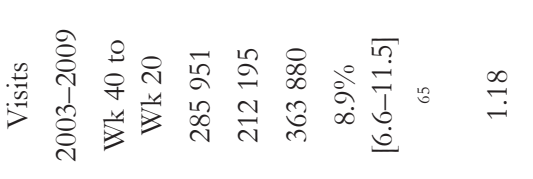

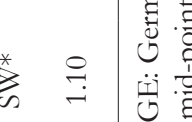

要

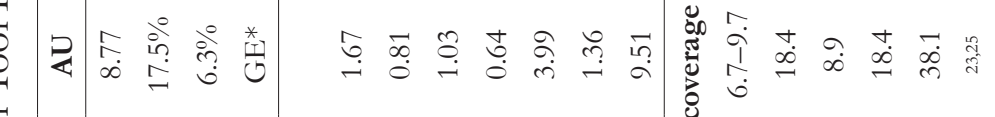

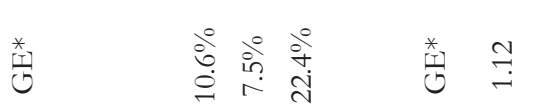

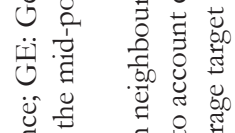

焉志

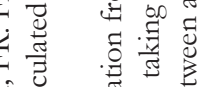

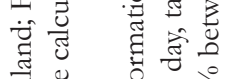

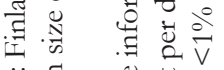

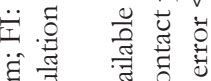

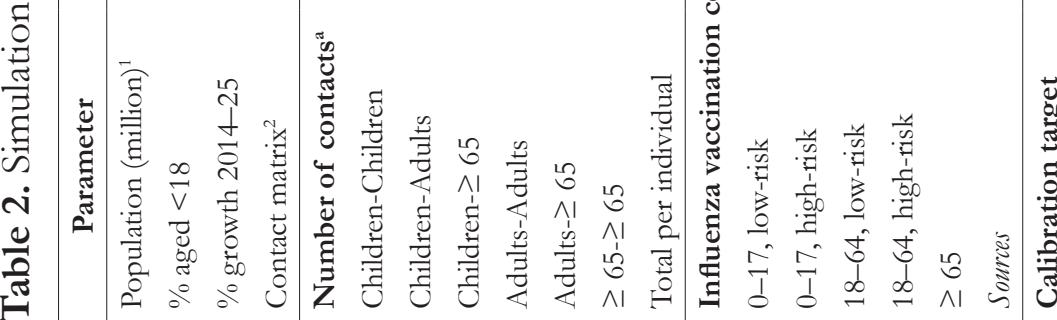

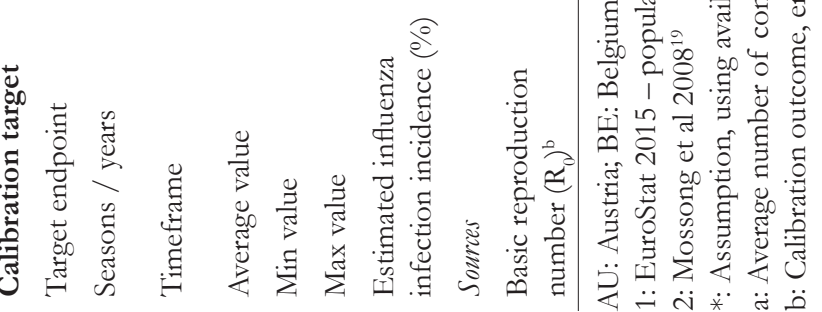




\section{Compared Vaccination Strategies}

The reference strategy was the unchanged country-specific coverage rate, using quadrivalent inactivated vaccine (QIV). In most European countries, influenza vaccination policies target only individuals with high risk from 6 months of age - with chronic disease/immune deficiency and/or aged individuals of $\geq 65$ years - representing approximately 180 million individuals $(36 \%)$ overall, in the European population. ${ }^{20}$ The proportion of high-risk individuals increased with age in the model: $16 \%$ of children (of whom $11 \%$ are ineligible to receive QLAIV due to a severe form of asthma $\left.{ }^{21}\right), 16 \%$ of $18-44$-year-olds, $32 \%$ of $18-64$-year-olds and $100 \%$ of those aged $\geq 65$ years by definition (Table 1). In the evaluated strategy of our simulations, children aged 5-10 years were additionnally vaccinated using QLAIV with a coverate rate of $56.8 \%$, as was the case in $2014-2015$ in the United Kingdom's primary school pilot areas. Children suffering from a severe form of asthma continued to receive QIV in our model, with new coverage of 56.8\%. Using the model, both strategies were evaluated during 10 influenza seasons, starting in 2015-2016. Current vaccination coverage rates per age-risk group and country were derived from the most recent reports by the European Centre for Disease Prevention and Control (ECDC), ${ }^{1,22}$ from multi-country surveys, ${ }^{23,24}$ and from country-specific studies ${ }^{13,25-38}$ (Table 2). According to a study by the French sick fund, ${ }^{39}$ individuals vaccinated in a given year had a higher probability of being revaccinated the following year (odds ratio [OR] 30-60). A preferential re-vaccination factor was implemented in the simulations accordingly for all age groups.

\section{Vaccination Properties}

The vaccine efficacy against influenza infection in children aged 2-17 years, assessed in meta-analyses of randomized controlled trials, was 59\% (95\% confidence interval [CI] [41-71\%]) for the trivalent inactivated vaccine and $80 \%\left[68-87 \%\right.$ ] for the trivalent live-attenuated vaccine. ${ }^{40}$ The trivalent inactivated vaccine showed an efficacy of $60 \%$ [53-66\%] in healthy adults, ${ }^{41}$ and $58 \%$ [34-73\%] in the population aged $>65$ years. ${ }^{42}$ The latter efficacy value was applied to all high-risk individuals using the inactivated vaccine. In the model, we assumed the efficacy of quadrivalent vaccines to be the same as that of the trivalent ones reported in the metaanalyses. ${ }^{43-45}$ The duration of vaccination-acquired immunity is known to wane quickly after vaccination with an inactivated vaccine; ${ }^{46,47}$ consequently, all QIV-acquired immunity was assumed to be lost after one influenza season. Immunity acquired by live-attenuated vaccination may last at least until the following season: according to an Asian study, $70 \%$ of the vaccinees who were successfully immunized in the first year with a live-attenuated vaccine were also protected in the second year against matched strains without re-vaccination. ${ }^{48}$ Accordingly, we assumed that $30 \%$ of the immunity acquired by QLAIV vaccination was lost at the end of the first influenza season, whereas the remaining part was lost after the second season (Table 1).

\section{Medical Resources}

Based on the simulated number of symptomatic influenza cases, the probability of requiring a primary care consultation (PCC), a hospitalization or an admission to the intensive care unit (ICU) was applied to estimate the total number of influenza-related medical resources used, with the reference and the evaluated strategies. Country-specific data regarding the management of influenza symptoms were consulted ${ }^{49-53}$ and high-level estimates of the medical resources probabilities were applied to all countries: the rate of PCC was assumed to be $33 \%$ in the general population and $67 \%$ for $0-1$ year-old children and high-risk individuals, the rate of hospital admission was assumed to be $0.2 \%$ in the general population, $1.0 \%$ for children aged $0-1$ years and high-risk individuals aged $2-64$ years and $3.0 \%$ for those aged $\geq 65$ years. Further, ICU admissions were assumed to occur in 10\% of hospitalization cases (Table 1). 


\section{Model Outcomes}

The number of symptomatic cases cumulated over the 10-year evaluation period was expressed as a number of cases per 100000 population-years (total number of events divided by the model time horizon ie, 10 years and by the average population size over the 10 -year evaluation period, then multiplied by 100000 ). The incidence was estimated in the total population and separately in the subgroup of children aged 5-10 years (targeted population) and in adults aged $\geq 18$ years. These calculations were applied to each country separately, and to the 13 countries pooled together.

The evaluated and reference strategies were then compared in terms of medical resources, using the same statistics as those calculated for the UK influenza season 2014-2015: OR for PCC in the target population (children aged $5-10$ years) and the non-targeted population of adults aged $\geq 18$ years, and numbers needed to vaccinate (NNV) with QLAIV to avert one PCC, one hospitalization or one ICU admission, respectively. The model outcomes were compared with published real-life data from the United Kingdom. ${ }^{8}$

\section{Sensitivity Analyses}

In a sensitivity analysis, the OR and NNV were estimated after varying the rate of medical resources used in case of symptomatic influenza (PCC, hospitalizations and ICU), using $\pm 25 \%$ variations around the base case probabilities.

Sensitivity analyses regarding the vaccine efficacy, basic reproduction number and immunity duration were performed elsewhere, based on the same version of the simulation model. ${ }^{18}$

\section{RESULTS}

In the following section and unless otherwise specified, the central value is obtained after cumulating the cases in 13 countries, and the ranges indicate the minimum and maximum values encountered for the 13 modelled countries.

\section{Impact on Symptomatic Cases}

When considering QLAIV vaccination coverage of 56.8\% of children aged 5-10 years, the absolute number of symptomatic influenza cases dropped from 205.2 (reference scenario) to 172.3 million (evaluated scenario) over 10 seasons in 13 countries ( $N=368.76$ million inhabitants on average over 2015-2025). This corresponds to 32.8 million prevented symptomatic influenza cases or 891 prevented cases per 100000 population-years in 13 European countries. Across countries, the number of symptomatic influenza cases of any age prevented by QLAIV vaccination ranged from 454 to 1663 cases per 100000 annually (lowest and highest values observed across 13 countries). Absolute numbers of cases per country are shown in Supplementary material S1.

\section{Odds Ratio Primary Care Consultation}

As a consequence of the prevented symptomatic cases, the number of influenza-related PCC cumulated over 10 years in the total population dropped from 86.3 million (2341 per 100000 population-years) to 72.9 million (1977 per 100000 population-years) ie, by 13.4 million (364 per 100000 population-years). In the target population of children aged 5-10 years, the reduction amounted to 115 PCC per 100000 populationyears (Table 3), ranging from 47 to 218 PCC per 100000 across 13 countries. The corresponding OR for PCC 
among 5-10 year-olds was 0.38 (Table 1) and ranged from 0.18 to 0.48 across the simulated countries, while the published OR in the United Kingdom was 0.06 with a 95\% CI of [0.01; 0.62] (Figure 1).

In adults aged $\geq 18$ years, the reduction was 189 PCC per 100000 population-years (Table 3 ), ranging from 98 to 359 PCC per 100000 across 13 countries. The ORs for PCC in adults was 0.89 (Table 3) and ranged from 0.60 to 0.91 (UK OR $[95 \% \mathrm{CI}]=0.41$ [0.19; 0.86]; Figure 2).

Table 3. PCC per 100,000 Population-years and OR with Evaluated versus Current Strategy, Model versus UK 2014-2015 Outcomes

\begin{tabular}{clccc}
\hline \multicolumn{1}{c}{ Age Group } & \multicolumn{1}{c}{ Outcome } & $\begin{array}{c}\text { Current } \\
\text { Strategy }\end{array}$ & $\begin{array}{c}\text { Evaluated } \\
\text { Strategy }\end{array}$ & $\begin{array}{c}\text { Odds Ratio } \\
\text { Evaluated vs Current }\end{array}$ \\
\hline \multirow{2}{*}{ 5-10 year-old (target) } & Rate (pooled 13 countries) & 185.7 & 70.6 & 0.38 \\
& Country range: minimum & 70.1 & 23.3 & 0.18 \\
& Country range: maximum & 324.0 & 135.8 & 0.48 \\
& UK 2014-2015 & 266.9 & 19.7 & $0.06[0.01 ; 0.62]$ \\
\hline \multirow{2}{*}{ Adults $\geq 18$ (non target) } & Rate (pooled 13 countries) & 1688.3 & 1499.7 & 0.89 \\
& Country range: minimum & 615.9 & 367.6 & 0.60 \\
& Country range: maximum & 2623.6 & 2398.2 & 0.91 \\
& UK 2014-2015 & 508.1 & 219.1 & $0.41[0.19 ; 0.86]$ \\
\hline
\end{tabular}

Current strategy: current vaccination coverage of high-risk individuals (country-specific rates), using QIV

Evaluated strategy: Current strategy + QLAIV in $56.8 \%$ of 5-10 year-old children (all countries)

UK 2014-2015: Current strategy = non pilot areas; Evaluated strategy $=$ 'primary school age children' area ${ }^{8}$

Rate per 100000 population-years calculated using the exposed population in the mid-point of the evaluation period and range (2015-2025)

PCC: primary care consultation

Figure 1. OR for PCC in Target Population (children aged 5-10 years), Model versus UK 2014-2015 Outcomes

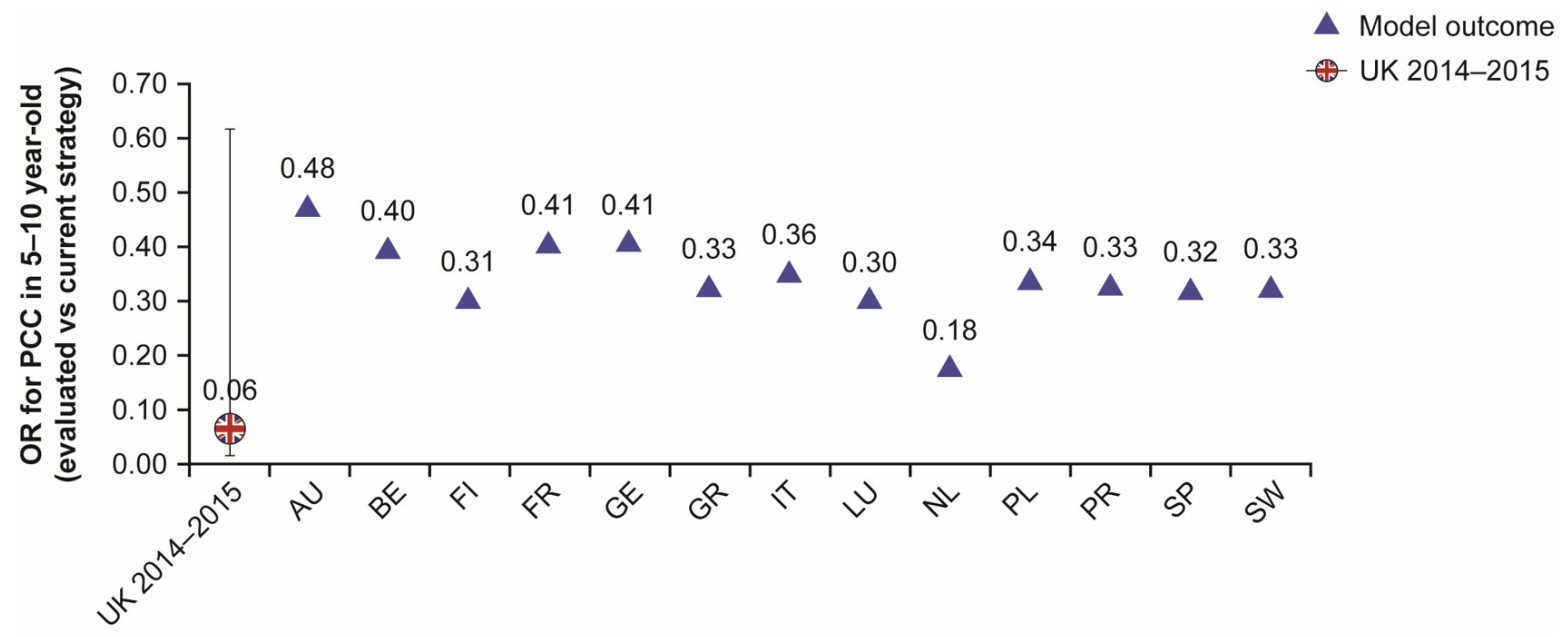

AU: Austria; BE: Belgium; FI: Finland; FR: France; GE: Germany; GR: Greece; IT: Italy; LU: Luxembourg; NL: The Netherlands; PL: Poland; PT: Portugal; SP: Spain; SW: Sweden; UK: United Kingdom

OR: odds ratio; PCC: primary care consultation

This chart shows the OR for PCC in the target population (children aged 5-10 years) modeled for 13 European countries, and compared to a similar outcome measured in the United Kingdom for season 2014-2015. The model-based OR for PCC in 5-10 yearold children ranged from 0.18 to 0.48 across 13 countries; this range falls within the $95 \%$ confidence interval of the UK results for season 2014-2015, which was [0.01-0.62]. 
Figure 2. OR for PCC in Non-target Population ( $\geq 18$ year-old), Model versus UK 2014-2015 Outcomes

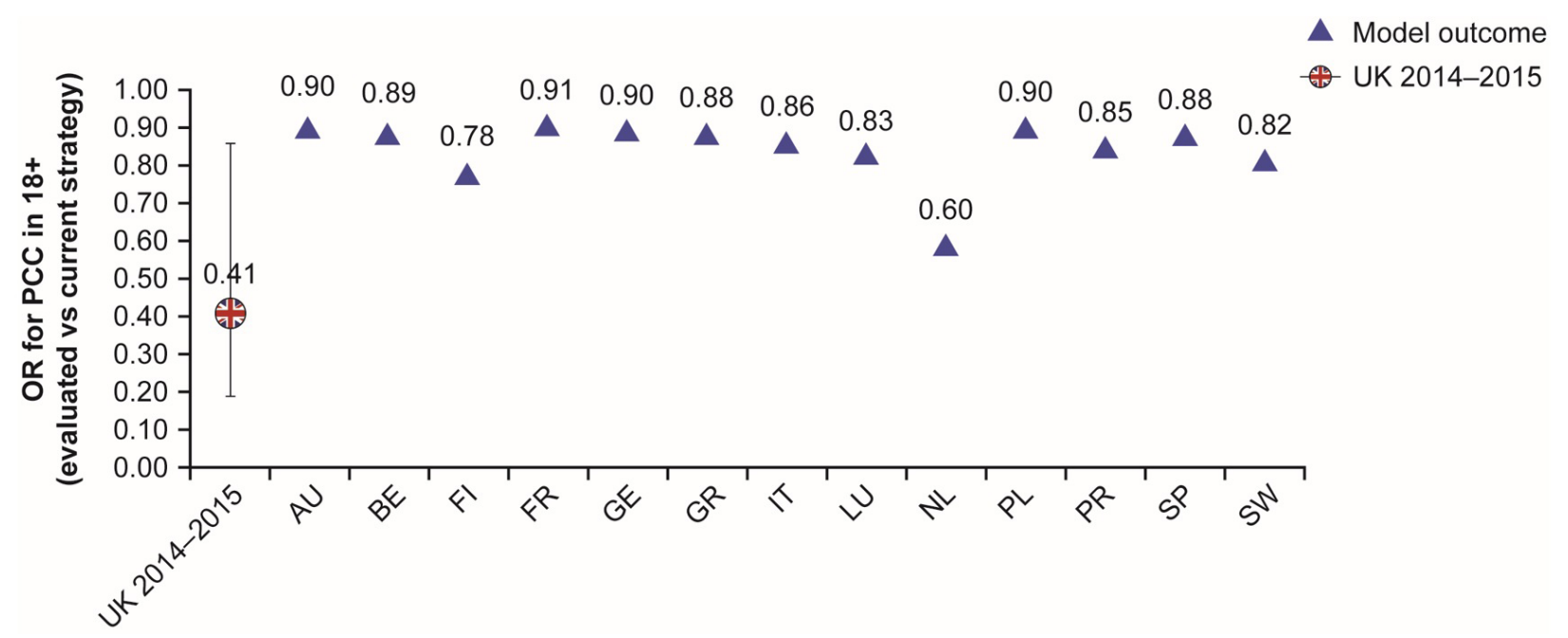

AU: Austria; BE: Belgium; FI: Finland; FR: France; GE: Germany; GR: Greece; IT: Italy; LU: Luxembourg; NL: The Netherlands; PL: Poland; PT: Portugal; SP: Spain; SW: Sweden; UK: United Kingdom

OR: odds ratio; PCC: primary care consultation

This chart shows the OR for PCC in the non-target population (adults aged 18 year-old and older) modeled for 13 European countries, and compared to a similar outcome measured in the United Kingdom for season 2014-2015. The model-based OR for PCC in adults ranged from 0.60 to 0.91 across 13 countries; this range overlaps within the $95 \%$ confidence interval of the UK results for season 2014-2015, which was [0.19-0.86].

\section{Number Needed to Vaccinate}

Across 13 countries and 10 years, the evaluated strategy required a total of 145.9 million QLAIV doses and prevented 13.4 million PCC overall. The number of QLAIV doses needed (NNV) per averted one PCC in the total population was 11, and ranged from 6 to 19 across 13 countries. The NNV reported in the UK analysis of season 2014-2015 was 16 per averted PCC (Table 4). In the model, cumulated over 10 years and 13 countries, 165064 influenza-related hospitalizations were prevented in the evaluated scenario. The NNV was 884 and ranged from 530 to 1524 per averted hospitalization (UK NNV=317). For ICU admissions, the NNV was 8838 and ranged from 5298 to 15241 per averted ICU admission (UK NNV=2205).

\section{Sensitivity Analyses}

A $50 \%$ reduction of the PCC probability in case of symptomatic influenza increased the NNV in our model to 22 and the country range to $12-37$. This scenario, assuming PCC rates of $17 \%$ in low risk individuals and $33 \%$ in 1 year-old children and high risk individuals, is still encompassing the observed UK NNV value for 2014-2015 (16 doses per averted PCC).

When increasing the base case PCC probability by 50\%, the model-based NNV decreased to seven (13-country range: 4-12).

For hospitalizations, the model-based NNV increased to 1768 (13-country range 1060-3048) when assuming hospitalization rates which were 50\% lower than in the base case. The NNV decreased to 589 (353-1016) when assuming 50\% higher hospitalization rates, which was closer to the observed UK NNV value for 2014-2015 (317 doses per averted hospitalization). 
For ICU, the model-based NNV increased to 17675 (13-country range 10 597-30 483) when assuming ICU rates which were 50\% lower than in the base case, and decreased to 5892 (3532-10 161) when assuming 50\% higher ICU rates. Both values were higher than the observed UK NNV value for 2014-2015 (2205 doses per averted ICU admission).

Varying the probabilities of medical resources used in case of symptomatic influenza only had a marginal impact on the ORs, because the targeted event (symptomatic influenza) remained rare (5563 cases per 100000 population-years in the reference strategy and 4673 per 100000 in the evaluated strategy).

Table 4. NNV with QLAIV per Prevented Event in the Total Population, Model versus UK 2014-2015 Outcomes

\begin{tabular}{llccc}
\hline \multicolumn{1}{c}{ Resource } & \multicolumn{1}{c}{ Outcome } & Current Strategy & Evaluated Strategy & Difference \\
\hline QLAIV doses & \multicolumn{1}{c}{ NCC } & 0 & 14.59 million & 14.59 million \\
\hline Pumber of cases & 86.34 million & 72.90 million & 13.50 million \\
& NNV (country range) & & & $11(6-19)$ \\
& UK 2014-2015 & & & 16 \\
\hline Hospitalization & Number of cases & 1.17 million & 1.00 million & 0.17 million \\
& NNV (country range) & & & $884(530-1524)$ \\
& UK 2014-2015 & & & 317 \\
\hline ICU & Number of cases & 116854 & & 100347 \\
& NNV (country range) & & & $8838(5298-15241)$ \\
& UK 2014-2015 & & & 2205 \\
\hline
\end{tabular}

Current strategy: current vaccination coverage of high-risk individuals (country-specific rates), using QIV Evaluated strategy: Current strategy + QLAIV in 56.8\% of 5-10 year-old children (all countries)

UK 2014-15: Current strategy $=$ non pilot areas; Evaluated strategy $=$ 'primary school age children' area ${ }^{8}$

Rate per 100000 population-years calculated using the exposed population in the mid-point of the evaluation period and range (2015-2025).

\section{DISCUSSION}

Across a range of European countries, our model showed beneficial impact of a paediatric vaccination programme using QLAIV in primary school age children, by reducing the number of symptomatic influenza cases both in the target vaccinated children and in adult community around them, the non-target populations. As a consequence, the medical resources used to manage symptomatic influenza, including PCCs and hospitalizations, were reduced in children and adults, consistent with what was observed during a single season in the United Kingdom. These findings show that translating the UK experience to other European countries would provide similar public health benefits from a paediatric vaccination programme, on top of the current strategies mainly targeting at risk groups.

Most of our model-based results were more conservative than the observed outcomes, especially the ORs in the non-target population (adults): the ORs for PCC in the adult population were distributed around the upper value of the UK 95\% CI (ie, 0.86, see Figure 2), ranging from 0.60 in The Netherlands to 0.91 in France. The comparatively conservative outcome of our simulation studies may - at least in part - be explained by the fact that we compare a 10-year evaluation period to a single influenza season: it has been reported that shortly after implementing a new vaccination strategy (as was the case in the United Kingdom), a combination of the pre-existing high level of natural immunity and the newly acquired vaccination-derived immunity lead to a 
transient period of over-optimistic results (termed as "honeymoon period" 54 ).

Our simulation studies also show a few years of high yields after introducing QLAIV vaccination which gradually decline to a more moderate long-term level.

The two factors which had the largest impact on our results (see previous sensitivity analyses done with the same model ${ }^{15-17}$ ) were the basic reproduction number $\mathrm{R}_{0}$ and the duration of naturally acquired immunity after influenza $A$ infection. The values of $\mathrm{R}_{0}$ were determined by calibration to observed incidence data from the 13 countries and were largely driven by the countries' demographic structures and the frequency of contacts between age groups. Similar results were obtained in a simulation study comparing influenza simulation results across countries ${ }^{55}$ ). At the country level, the largest and smallest model-based OR for PCC were observed in the countries with, respectively, the smallest and the largest values of all-year average $\mathrm{R}_{0}: 0.90$ in The Netherlands (winter peak value: 1.29 ) and 1.28 in France (peak: 1.83).

We used high-level, simple, probabilities of medical resources used in case of symptomatic influenza, as our purpose was to raise awareness around the potential benefits of a UK-like paediatric programme across a range of demographic features, contact patterns, current vaccination coverage and local influenza incidence. Further heterogeneity concerning the influenza-related PCC or hospitalization rates was not specified by country in the current analysis. Across the country-specific sources that we consulted to assess influenza-related medical resources use, there were indeed important variations in study design (surveillance networks data, administrative/ medical records, or prospective observational studies) and reporting (for example, by age and/or risk status or overall, by vaccination status or not, during a full season or only during peak influenza activity).

Our goal was to present an overall European picture of the QLAIV vaccination effect rather than a betweencountries comparison. For this reason, no further comparisons or country-level interpretations were undertaken and additional, local medico-economic data would need to be collected to further investigate the return on investment of the evaluated program in a given country. In particular, some countries like The Netherlands or Sweden are taking into account not only the direct medical resources associated with influenza, but also the work productivity losses, to estimate the societal impact of preventing symptomatic cases. Lost work days caused by symptomatic influenza are indeed responsible for a huge economic burden every year in Europe E $^{20}$ and this was not included in our study.

Important differences between the healthcare system in the United Kingdom and the systems across the 13 European countries represent a limitation of our comparison. For both medical resource and local data, a degree of pragmatism must be employed when assessing the level of granularity of data in the model.

Another limitation concerns the different PCC definitions used: the reported primary care consultations in the UK study include influenza-like illness whereas our model only counts PCC from confirmed influenza cases. This means that the baseline (events occurring in the 'reference' group with current vaccination strategy) was including more heterogeneous events in the UK study compared to our model. This difference in PCC definitions might impact the relative effect of the evaluated vaccination strategy versus baseline. For hospitalization and ICU, the UK 'real-life' study used the same 'confirmed influenza' definition as our simulation study. Modelbased NNV appeared even more conservative compared with PCC, which indicates that our conclusions are robust to changes in influenza-event definitions.

In the Cochrane meta-analysis providing paediatric vaccine efficacy data for the model, the QLAIV efficacy against influenza infection in children was $80 \%$ (risk ratio 0.20 [0.13;0.32]) which was based on six studies 
with 9175 participants. In recent seasons a lower QLAIV effectiveness against influenza A/H1N1pdm09 was observed in the United States ${ }^{56}$ while the effectiveness of the live vaccine was found similar to that of the inactivated vaccine in a Canadian study. ${ }^{57}$ In view of these conflicting observations, extensive investigations are currently under way to gain an in-depth understanding of the live vaccine's efficacy and effectiveness. In the model, a reduced efficacy for QLAIV would lead to a lower number of averted influenza cases; however, the incremental benefit of extending the coverage of children would remain positive, as seen in previously published sensitivity analyses with the model. ${ }^{16,17}$

Based on the currently available evidence, our study shows that the vaccination of a large group of primary school age children with QLAIV in Europe could generate substantial benefits in the vaccinated paediatric population, and reduce the medical resources use in the adult population as well. These direct and indirect benefits of paediatric infuenza vaccination were observed in the United Kingdom during season 2014-2015, and our model-based predictions across 13 European countries compared favorably to the real-life UK data.

\section{FINANCIAL SUPPORT}

This study was funded by an unrestricted grant from AstraZeneca.

\section{CONFLICT OF INTEREST}

JH, RL and SDSM are or were employees of AstraZeneca at the time of the study. LG is an employee of QuintilesIMS which has received consulting fees from AstraZeneca. ME is a partner and shareholder of the contract research and consulting institute Epimos $\mathrm{GmbH}$, which has received consulting fees and research support from AstraZeneca, Novartis, and GlaxoSmithKline.

\section{AUTHORSHIP}

ME conceptualized the study, carried out the simulations and interpreted the results. LG provided local data inputs, analysed the simulation results, and drafted the manuscript. JH, RL and SDSM provided expertise and guidance on data input and assumptions. All authors critically appraised, corrected and approved the manuscript before submission.

\section{REFERENCES}

1 Mereckiene J, Cotter S, Nicoll A, et al: Seasonal influenza immunisation in Europe. Overview of recommendations and vaccination coverage for three seasons: pre-pandemic (2008/09), pandemic (2009/10) and post-pandemic (2010/11). Euro Surveill 2014;19:20780.

${ }^{2}$ Kostova D, Reed C, Finelli L, et al: Influenza Illness and Hospitalizations Averted by Influenza Vaccination in the United States, 2005-2011. PLoS One 2013;8:e66312.

${ }^{3}$ Kwong JC, Stukel TA, Lim J, et al: The effect of universal influenza immunization on mortality and health care use. PLoS Med 2008;5:e211.

${ }^{4}$ Reichert TA, Sugaya N, Fedson DS, et al: The Japanese experience with vaccinating schoolchildren against influenza. N Engl J Med 2001;344:889-896.

${ }^{5}$ Piedra PA, Gaglani MJ, Kozinetz CA, et al: Herd immunity in adults against influenza-related illnesses with use of the trivalent-live attenuated influenza vaccine (CAIV-T) in children. Vaccine 2005;23:1540-1548. 
${ }^{6}$ Glezen WP, Gaglani MJ, Kozinetz CA, et al: Direct and indirect effectiveness of influenza vaccination delivered to children at school preceding an epidemic caused by 3 new influenza virus variants. J Infect Dis 2010;202:1626-1633.

${ }^{7}$ Loeb M, Russell ML, Moss L, et al: Effect of influenza vaccination of children on infection rates in Hutterite communities: a randomized trial. JAMA 2010;303:943-950.

${ }^{8}$ Pebody RG, Green HK, Andrews N, et al: Uptake and impact of vaccinating school age children against influenza during a season with circulation of drifted influenza A and B strains, England, 2014/15. Euro Surveill 2015;20(39).

${ }^{9}$ Jordan R, Connock M, Albon E, et al: Universal vaccination of children against influenza: are there indirect benefits to the community? A systematic review of the evidence. Vaccine 2006;24:1047-1062.

${ }^{10}$ Weycker D, Edelsberg J, Halloran ME, et al: Population-wide benefits of routine vaccination of children against influenza. Vaccine 2005;23:1284-1293.

${ }^{11}$ Vynnycky E, Pitman R, Siddiqui R, et al: Estimating the impact of childhood influenza vaccination programmes in England and Wales. Vaccine 2008;26:5321-5330.

${ }^{12}$ Pitman RJ, White LJ, Sculpher M: Estimating the clinical impact of introducing paediatric influenza vaccination in England and Wales. Vaccine 2012;30:1208-1224.

13 Baguelin M, Flasche S, Camacho A, et al: Assessing optimal target populations for influenza vaccination programmes: an evidence synthesis and modelling study. PLoS Med 2013;10:e1001527.

${ }^{14}$ Rose MA, Damm O, Greiner W, et al: The epidemiological impact of childhood influenza vaccination using live-attenuated influenza vaccine (LAIV) in Germany: predictions of a simulation study. BMC Infect Dis 2014;14:40.

${ }^{15}$ Damm O, Eichner M, Rose MA, et al: Public health impact and cost-effectiveness of intranasal live attenuated influenza vaccination of children in Germany. Eur J Health Econ 2015;16:471-488.

${ }^{16}$ Gerlier L, Lamotte M, Dos Santos Mendes S, et al: Estimates of the Public Health Impact of a Pediatric Vaccination Program Using an Intranasal Tetravalent Live-Attenuated Influenza Vaccine in Belgium. Paediatr Drugs 2016;18:303-318.

${ }^{17}$ Gerlier L, Lamotte M, Grenèche S, et al: Assessment of public health and economic impact of intranasal live-attenuated influenza vaccination of children in France using a dynamic transmission model. Appl Health Econ Health Policy 2017;15:261-276.

${ }^{18}$ Gerlier L, Hackett J, Lawson R, et al: Direct and indirect protection with paediatric influenza vaccination in Europe estimated by a dynamic transmission model. OPTIONS IX Control Infu Chicago, Illinois, USA 2016. p. O43.

${ }^{19}$ Mossong J, Hens N, Jit M, et al: Social contacts and mixing patterns relevant to the spread of infectious diseases. PLoS Med 2008;5:e74.

${ }^{20}$ Preaud E, Durand L, Macabeo B, et al: Annual public health and economic benefits of seasonal influenza vaccination: a European estimate. BMC Public Health 2014;14:813.

${ }^{21}$ Weil-Olivier C, Lina B: Vaccination coverage with seasonal and pandemic influenza vaccines in children in France, 2009-2010 season. Vaccine 2011;29:7075-7079.

${ }^{22}$ European Centre for Disease Control and Prevention: Implementation of the Council Recommendation on seasonal influenza vaccination (2009/1019/EU). https://ecdc.europa.eu/sites/portal/files/media/ en/publications/Publications/Implementation-seasonal-influenza-vaccination-Council-RecommendationJan-2014.pdf. Accessed November 1, 2016. 
${ }^{23}$ Blank PR, Schwenkglenks M, Szucs TD: Vaccination coverage rates in eleven European countries during two consecutive influenza seasons. J Infect 2009;58:446-458.

${ }^{24}$ Influenzanet: Influenzanet: a network of European citizens fighting against influenza. Available from: https:// www.influenzanet.eu/en/ Accessed March 29, 2016.

${ }^{25}$ Kunze U, Bohm G, Groman E: Influenza vaccination in Austria from 1982 to 2011: a country resistant to influenza prevention and control. Vaccine 2013;31:5099-5103.

${ }^{26}$ ISP/WIV: HISIA : Belgian Health Interview Survey - Interactive Analysis. Available from: https://hisia.wivisp.be/SitePages/Home.aspx Accessed March 27, 2016.

${ }^{27}$ Tuppin P, Samson S, Weill A, et al: Seasonal influenza vaccination coverage in France during two influenza seasons (2007 and 2008) and during a context of pandemic influenza A(H1N1) in 2009. Vaccine 2011;29:46324637.

${ }^{28}$ Groupe d'Expertise et d'Information sur la Grippe (GEIG): Le taux de couverture vaccinale en France. Last updated. Available from: http://www.grippe-geig.com/couverture-vaccinale.html. Accessed March 27, 2016.

${ }^{29}$ Schröder-Bernhardi D: Schutzimpfungen gegen Influenza werden bei Kindern und Jugendlichen äußerst selten durchgeführt - Ergebnisse einer Datenbankanalyse. 2014. In press.

${ }^{30}$ Bodeker B, Remschmidt C, Schmich P, et al: Why are older adults and individuals with underlying chronic diseases in Germany not vaccinated against flu? A population-based study. BMC Public Health 2015;15:618.

${ }^{31}$ Mamma M, Spandidos DA: Economic evaluation of the vaccination program against seasonal and pandemic A/H1N1 influenza among customs officers in Greece. Health Policy 2013;109:71-77.

32 Krippler S: Enquête de couverture vaccinale au Grand-Duché de Luxembourg 2012. 2014. In press.

33 Tacken M, Jansen B, Mulder J, et al: Monitoring vaccinatiegraad Nationaal Programma Grieppreventie 2013. Available from: http://www.rivm.nl/Documenten_en_publicaties/Algemeen_Actueel/Uitgaven/ Preventie_Ziekte_Zorg/Griepprik/Monitoring_vaccinatiegraad_Nationaal_Programma_Grieppreventie_ NPG. Accessed June 6, 2017.

${ }^{34}$ Brydak LB, Wozniak Kosek A, Nitsch-Osuch A: Influenza vaccines and vaccinations in Poland - past, present and future. Med Sci Monit 2012;18:RA166-171.

${ }^{35}$ Nunes B, Sousa Uva M, Roquette R, et al: Vacinação antigripal da população portuguesa na época 2013-2014 - Estudo na amostra ECOS. Available from: http://www.ulsm.min-saude.pt/ResourcesUser/Documentos/ Vacinacao_antigripal_da_populacao_portuguesa_na_epoca_2013_2014.pdf. Accessed June 6, 2017.

${ }^{36}$ Jimenez-Garcia R, Esteban-Vasallo MD, Rodriguez-Rieiro C, et al: Coverage and predictors of vaccination against 2012/13 seasonal influenza in Madrid, Spain: analysis of population-based computerized immunization registries and clinical records. Hum Vaccin Immunother 2014;10:449-455.

${ }^{37}$ Public Health Agency of Sweden: Influenza in Sweden Season 2013-2014. Available from: https://www. folkhalsomyndigheten.se/pagefiles/18481/Influenza-in-Sweden-2013-2014.pdf. Accessed June 6, 2017.

${ }^{38}$ Public Health England: Seasonal influenza vaccine uptake amongst GP patients in England. Available from: https://www.gov.uk/government/statistics/seasonal-flu-vaccine-uptake-in-gp-patients-in-england-winterseason-2014-to-2015. Accessed June 6, 2017.

39 Tuppin P, Samson S, Weill A, et al: Influenza vaccination coverage in France in 2007-2008: contribution of vaccination refund data from the general health insurance scheme]. Med Mal Infect 2009;39:780-788.

${ }^{40}$ Jefferson T, Rivetti A, Di Pietrantonj C, et al: Vaccines for preventing influenza in healthy children. Cochrane Database Syst Rev 2012:CD004879. 
${ }^{41}$ Jefferson T, Di Pietrantonj C, Rivetti A, et al: Vaccines for preventing influenza in healthy adults. Cocbrane Database Syst Rev 2014:CD001269.

${ }^{42}$ Jefferson T, Di Pietrantonj C, Al-Ansary LA, et al: Vaccines for preventing influenza in the elderly. Cochrane Database Syst Rev 2010:CD004876.

${ }^{43}$ Block SL, Falloon J, Hirschfield JA, et al: Immunogenicity and safety of a quadrivalent live attenuated influenza vaccine in children. Pediatr Infect Dis J 2012;31:745-751.

${ }^{44}$ Block SL, Yi T, Sheldon E, et al: A randomized, double-blind noninferiority study of quadrivalent live attenuated influenza vaccine in adults. Vaccine 2011;29:9391-9397.

${ }^{45}$ Kieninger D, Sheldon E, Lin WY, et al: Immunogenicity, reactogenicity and safety of an inactivated quadrivalent influenza vaccine candidate versus inactivated trivalent influenza vaccine: a phase III, randomized trial in adults aged $>/=18$ years. BMC Infect Dis 2013;13:343.

${ }^{46}$ Ambrose CS, Wu X, Belshe RB: The efficacy of live attenuated and inactivated influenza vaccines in children as a function of time postvaccination. Pediatr Infect Dis J 2010;29:806-811.

${ }^{47}$ Belongia EA, Sundaram ME, McClure DL, et al: Waning vaccine protection against influenza A (H3N2) illness in children and older adults during a single season. Vaccine 2015;33:246-251.

${ }^{48}$ Tam JS, Capeding MR, Lum LC, et al: Efficacy and safety of a live attenuated, cold-adapted influenza vaccine, trivalent against culture-confirmed influenza in young children in Asia. Pediatr Infect Dis J 2007;26:619-628.

49 Grippenet fr: Bilan de la saison 2014-2015. https://www.grippenet.fr/media/uploads/bilan_ saison_2014_2015.pdf. Accessed May 30, 2016.

${ }^{50}$ Beutels P, Vandendijck Y, Willem L, et al: Seasonal influenza vaccination: prioritizing children or other target groups? Part II: cost-effectiveness analysis. Health Technology Assessment (HTA) Brussels: Belgian Health Care Knowledge Centre (KCE). 2013. KCE Reports 204. D/2013/10.273/43. https://kce.fgov.be/sites/ default/files/page_documents/KCE_204_Seasonal_influenza_vaccination_partII.pdf. Accessed March 29, 2016.

${ }^{51}$ Institut National de Veille Sanitaire (INVS): BEH - Bulletin épidémiologique hebdomadaire n.d. Last updated. http:/ /invs.santepubliquefrance.fr//Publications-et-outils/BEH-Bulletin-epidemiologique-hebdomadaire. Accessed March 29, 2016.

${ }^{52}$ Bilcke J, Coenen S, Beutels P: Influenza-like-illness and clinically diagnosed flu: disease burden, costs and quality of life for patients seeking ambulatory care or no professional care at all. PLoS One 2014;9:e102634.

${ }^{53}$ Riquelme R, Torres A, Rioseco ML, et al: Influenza pneumonia: a comparison between seasonal influenza virus and the H1N1 pandemic. Eur Respir J 2011;38:106-111.

${ }^{54}$ Scherer A, McLean A: Mathematical models of vaccination. Br Med Bull 2002;62:187-199.

${ }^{55}$ Schmidt SA, Kahlert J, Vestergaard M, et al: Hospital-based herpes zoster diagnoses in Denmark: rate, patient characteristics, and all-cause mortality. BMC Infect Dis 2016;16:99.

${ }^{56}$ Flannery B, Chung J: Influenza Vaccine Effectiveness, Including LAIV vs IIV in Children and Adolescents, US Flu VE Network, 2015-16. Available from: https://www.cdc.gov/vaccines/acip/meetings/downloads/ slides-2016-06/influenza-05-flannery.pdf. Accessed March 29, 2016.

${ }^{57}$ Loeb M, Russell ML, Manning V, et al: Live Attenuated Versus Inactivated Influenza Vaccine in Hutterite Children: A Cluster Randomized Blinded Trial. Ann Intern Med 2016;165:617-624.

${ }^{58}$ European Centre for Disease Control and Prevention: Guidance - Priority Risk Groups For Influenza Vaccination. Available from: http://ecdc.europa.eu/en/publications/Publications/0808_GUI_Priority_ Risk_Groups_for_Influenza_Vaccination.pdf. Accessed June 6, 2017. 
${ }^{59}$ Carrat F, Vergu E, Ferguson NM, et al: Time lines of infection and disease in human influenza: a review of volunteer challenge studies. Am J Epidemiol 2008;167:775-785.

${ }^{60}$ Williams CJ, Schweiger B, Diner G, et al: Seasonal influenza risk in hospital healthcare workers is more strongly associated with household than occupational exposures: results from a prospective cohort study in Berlin, Germany, 2006/07. BMC Infect Dis 2010;10:8.

${ }^{61}$ Swedish Institute for Communicable Disease Control. Influenza in Sweden Season 2012-2013. Available from: https://www.folkhalsomyndigheten.se/pagefiles/15027/influenza-in-sweden-2011-2012.pdf. Accessed March 29, 2016.

${ }^{62}$ Gasparini R, Bonanni P, Amicizia D, et al: Influenza epidemiology in Italy two years after the 2009-2010 pandemic: need to improve vaccination coverage. Hum Vaccin Immunother 2013;9:561-567.

${ }^{63}$ McDonald SA, Presanis AM, De Angelis D, et al: An evidence synthesis approach to estimating the incidence of seasonal influenza in the Netherlands. Influenza Other Respir Viruses 2014;8:33-41.

${ }^{64}$ Kovacs G, Kalo Z, Jahnz-Rozyk K, et al: Medical and economic burden of influenza in the elderly population in central and eastern European countries. Hum Vaccin Immunother 2014;10:428-440.

${ }^{65}$ Hanquet G, Jonckheer P, Vlayen J, et al: Seasonal influenza vaccination: priority target groups - Part 1. Good Clinical Practice (GCP). KCE Reports 162C. https://kce.fgov.be/sites/default/files/page_documents/ kce_162c_seasonal_influenza_vaccination.pdf. Accessed June 6, 2017.

${ }^{66}$ Nunes B, Viboud C, Machado A, et al: Excess mortality associated with influenza epidemics in Portugal, 1980 to 2004. PLoS One 2011;6:e20661.

${ }^{67}$ Larrauri Camara A, Jimenez-Jorge S, Mateo Ontanon S, et al: Epidemiology of the 2009 influenza pandemic in Spain. The Spanish Influenza Surveillance System. Enferm Infecc Microbiol Clin 2012;30 Suppl 4:2-9.

${ }^{68}$ Tuppin P, Choukroun S, Samson S, et al: [Vaccination against seasonal influenza in France in 2010 and 2011: decrease of coverage rates and associated factors]. Presse Med 2012;41:e568-576.

${ }^{69}$ Lytras T, Kossyvakis A, Melidou A, et al: Influenza vaccine effectiveness against laboratory confirmed influenza in Greece during the 2013-2014 season: a test-negative study. Vaccine 2015;33:367-373. 